\title{
Article \\ Silicon-Based All-Dielectric Metasurface on an Iron Garnet Film for Efficient Magneto-Optical Light Modulation in Near IR Range
}

\author{
Denis M. Krichevsky ${ }^{1,2,3, *}$, Shuang Xia ${ }^{4,5}$, Mikhail P. Mandrik ${ }^{6}$, Daria O. Ignatyeva ${ }^{2,3,7}$ (D, Lei Bi ${ }^{4,5}$ \\ and Vladimir I. Belotelov 2,3,7 (1)
}

check for

updates

Citation: Krichevsky, D.M.; Xia, S.; Mandrik, M.P.; Ignatyeva, D.O.; Bi, L.; Belotelov, V.I. Silicon-Based

All-Dielectric Metasurface on an Iron Garnet Film for Efficient

Magneto-Optical Light Modulation in Near IR Range. Nanomaterials 2021, 11, 2926. https://doi.org/10.3390/ nano11112926

Academic Editor: Daria A. Smirnova

Received: 7 October 2021

Accepted: 28 October 2021

Published: 1 November 2021

Publisher's Note: MDPI stays neutral with regard to jurisdictional claims in published maps and institutional affiliations.

Copyright: (c) 2021 by the authors. Licensee MDPI, Basel, Switzerland. This article is an open access article distributed under the terms and conditions of the Creative Commons Attribution (CC BY) license (https:/ / creativecommons.org/licenses/by/ $4.0 /)$.
1 Moscow Institute of Physics and Technology (MIPT), 141700 Dolgoprudny, Russia

2 Russian Quantum Center, 121353 Moscow, Russia; daria.ignatyeva@gmail.com (D.O.I.); belotelov@physics.msu.ru (V.I.B.)

3 Physics and Technology Institute, Vernadsky Crimean Federal University, 295007 Simferopol, Russia

4 National Engineering Research Center of Electromagnetic Radiation Control Materials, University of Electronic Science and Technology of China, Chengdu 610054, China; xiashuang_1996@163.com (S.X.); bilei@mit.edu (L.B.)

5 State Key Laboratory of Electronic Thin-Films and Integrated Devices, University of Electronic Science and Technology of China, Chengdu 610054, China

6 Faculty of Fundamental Physical and Chemical Engineering, Lomonosov Moscow State University, 119991 Moscow, Russia; skyworker14@yandex.ru

7 Photonic and Quantum Technologies School, Lomonosov Moscow State University, 119991 Moscow, Russia

* Correspondence: krichevskii.dm@phystech.edu

\begin{abstract}
All-dielectric nanostructures provide a unique low-loss platform for efficiently increasing light-matter interaction via excitation of the localized or propagating optical modes. Here, we report on the transverse magneto-optical Kerr effect enhancement in an all-dielectric metasurface based on a two-dimensional array of Si nanodisks on a cerium substituted dysprosium iron garnet thin film. We observed up to $15 \%$ light intensity modulation under TM modes excitation. The observed magneto-optical effect is nearly independent of the rotation of the light incidence plane with respect to the metasurface. Being compatible with conventional semiconductor technology, our structure holds promise for device applications, such as light modulators, magnetic and chemical sensors.
\end{abstract}

Keywords: magneto-optics; dielectric nanophotonics; transverse magnetooptical Kerr effect

\section{Introduction}

Light manipulation via nanostructured materials is a prominent issue of modern nanophotonics. The advances of nanophotonics are currently applied in various technical fields, such as information processing [1-6], physical and chemical sensing [7-11], and quantum simulations [12]. Magnetic materials provide additional degree of freedom for light control via various magneto-optical effects, such as Faraday, Voight, transverse and polar Kerr effects [13]. Excitation of various optical modes, such as surface or localized plasmons [14-21], waveguiding modes [22-26], surface Tamm states, and cavity modes in photonic crystals $[9,10,27,28]$ strongly enhance the aforementioned effects in magnetic nanostructures. Metal nanostructures produce a notable field localization and enhancement in the metal based magnetic nanostructures. At the same time, light-matter interaction in such systems is always accompanied by high optical losses and heating, making them less suitable for device applications. At this point, all-dielectric nanostructures are very promising since they are substantially less dissipative.

Dielectric iron oxides, for instance, iron garnet thin films and crystals, are widely used in magneto-optical devices. Magneto-optical properties of these compounds strongly depend on elemental composition [13]. All-dielectric iron-garnet nanogratings were reported 
to boost the magneto-optical response [22,23]. However, the fabrication of iron garnetbased nanostructures, such as one- or two-dimensional gratings, is a complicated process that is typically accompanied by focused ion beam (FIB) technology [22,23]. A dielectric grating is used to couple light with matter and excite optical modes. For these purposes, widely used semiconductor materials can be utilized, such as GaP, GaAs, InP, and Si. The latter material has a well-developed technological process of deposition, processing, and nanofabrication. Si-based nanostructures are currently used in a variety of applications, including chemical sensing [29], holography [30], flat optics [31], and data processing via light control (light wavelength, polarization state, transmission, and reflectivity) [32]. The mentioned technological advances make the combination of Si nanostructures with iron-garnet films an excellent candidate for enhanced magneto-optics.

In this paper, we report on an enhanced magneto-optical response observed in the all-dielectric structure based on a two-dimensional (2D) grating of Si nanodisks on a cerium substituted dysprosium iron garnet thin film in the near IR range. The periodicity of grating allows the excitation of the guided modes in the magnetic layer, which mediates a resonant increase of the transverse magneto-optical Kerr effect (TMOKE). TMOKE amplitude and spectral position are shown to be almost independent of the sample rotation around its normal. This feature, combined with the ease of fabrication process, makes the structure promising for applications in sensing and magnetometry.

\section{Materials and Methods}

\subsection{Samples Fabrication}

Pulsed laser deposition (PLD) was used to grow a $150 \mathrm{~nm}$ thick cerium substituted dysprosium iron garnet thin film of composition $\left(\mathrm{Ce}_{1} \mathrm{Dy}_{2}\right)\left(\mathrm{Al}_{0.42} \mathrm{Fe}_{4.58}\right) \mathrm{O}_{12}$ (Ce:DyIG) with a $50 \mathrm{~nm}$ thick yttrium iron garnet (YIG) layer on a fused quartz substrate. The targets were ablated with a $10 \mathrm{~Hz}, 248 \mathrm{~nm} \mathrm{KrF}$ excimer laser. The 50-nm-thick YIG film was first deposited on the silica substrate and served as a seed layer to promote the crystallization of the upper Ce:DyIG film. The substrate temperature was $400{ }^{\circ} \mathrm{C}$ and oxygen pressure was 10 mTorr during the YIG deposition process. The film was then rapidly annealed for $480 \mathrm{~s}$ at $900{ }^{\circ} \mathrm{C}$ and 80 Torr oxygen pressure. The aluminum-doped 150-nm-thick Ce:DyIG was deposited by exchanging targets of $\mathrm{Ce}_{1} \mathrm{Dy}_{2} \mathrm{Fe}_{5} \mathrm{O}_{12}$ and $\mathrm{Ce}_{1} \mathrm{Dy}_{2} \mathrm{Al}_{1} \mathrm{Fe}_{4} \mathrm{O}_{12}$ at the substrate temperature of $750{ }^{\circ} \mathrm{C}$ and an oxygen pressure of $5 \mathrm{mTorr}$.

Following the deposition of the magneto-optical films, an amorphous silicon thin film of $120 \mathrm{~nm}$ thickness was grown via plasma-enhanced chemical vapor deposition (PECVD). The patterns of a negative electron-beam resist HSQ were then exposed using electron beam lithography (EBL). Following that, a two-dimensional array of the Si nanodisks of $170 \mathrm{~nm}$ radius was fabricated using reactive ion etching (RIE) with HSQ as the resist. The Si nanodisks form a grating with a square lattice and a $500 \mathrm{~nm}$ period (Figure 1).

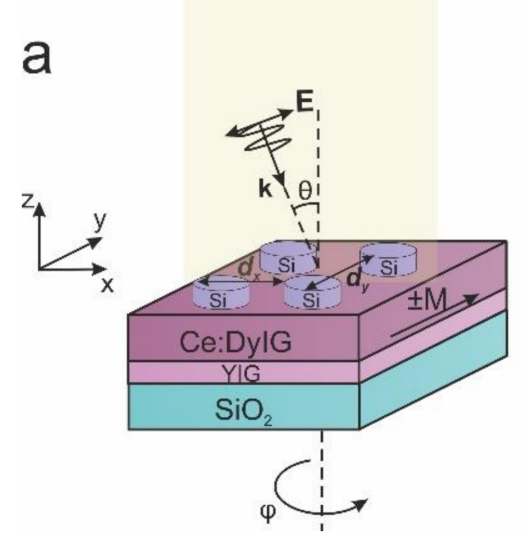

b

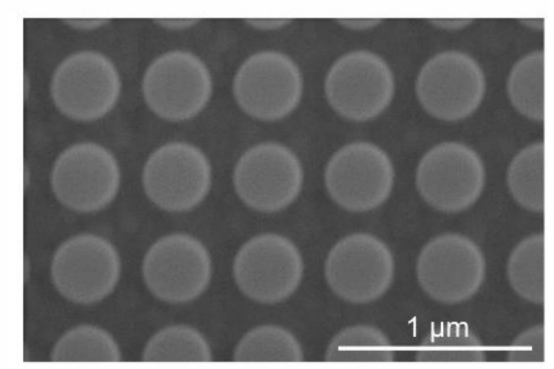

Figure 1. Schematic representation of the magneto-optical metasurface of Si nanodisk array on a Ce:DyIG (a) and SEM image of the sample (b). 


\subsection{TMOKE Measurements}

The angle-resolved transmittance and TMOKE spectra were measured using a Fourier experimental setup. The light from a halogen lamp (spectral range from 360 to $2500 \mathrm{~nm}$ ) was collimated with a lens (focal length $35 \mathrm{~mm}$ ) and polarized with a Glan-Taylor prism. Linearly polarized light after the Glan-Taylor prism was focused on the sample using a $20 \times$ microscope objective. The p-polarized (polarized in the incidence plane) light passed through the sample was collimated on the spectrometer by another $20 \times$ microscope objective and a system of lenses with focal length $300 \mathrm{~mm}$ and $150 \mathrm{~mm}$. The transmittance was obtained by comparing the sample's spectrum to the spectrum of the light source. For angle-resolved TMOKE spectra measurements, the sample was placed in a uniform magnetic field of $100 \mathrm{mT}$ generated by an electromagnet in transversal configuration (see Figure 1a). TMOKE dependence on the azimuth angle was also measured by rotating the sample around its normal. In this case, the transversal configuration of the magnetic field with respect to the light incident plane was preserved. The scheme of the setup is presented in Appendix D (see, Figure A4).

\subsection{Numerical Simulation}

The rigorous coupled-wave analysis (RCWA) approach was utilized for electromagnetic numerical simulation of the structure's optical and magneto-optical properties [33,34]. For simplicity, Dy:CeIG and YIG layers were substituted by a single $200 \mathrm{~nm}$ thick magnetic layer with the components of dielectric permittivity tensor given in Appendix A (Figure A1). It was deduced from the optical spectra of the magnetic sample without $\mathrm{Si}$ nanodisks. The dielectric permittivity of Si was taken from reference [35]. The dielectric permittivity of the glass substrate was 2.10 .

\section{Results and Discussions}

\subsection{Optical Modes}

Experimentally measured transmission spectra of the sample contain a number of well-defined resonances (Figure 2a). The transmission spectrum of the sample exhibits pronounced dips at $\sim 985,935,828$, and $768 \mathrm{~nm}$ at normal incidence of light. The resonances at 935 and $768 \mathrm{~nm}$ possess clear V-shaped angle-dependent evolution behavior as the polar incident angle $(\theta)$ increases. Simultaneously, resonances at $985 \mathrm{~nm}$ and $828 \mathrm{~nm}$ are almost independent of the polar angle.

a

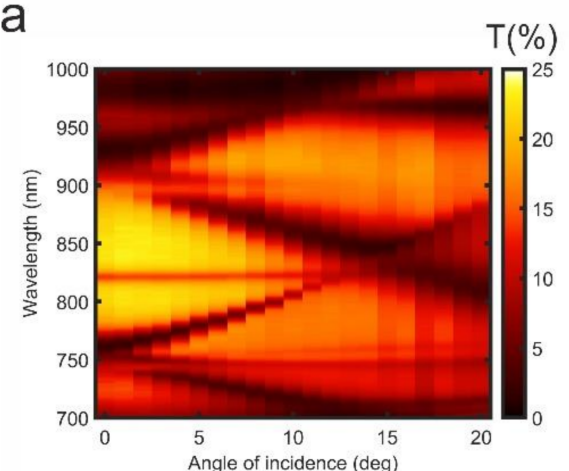

b

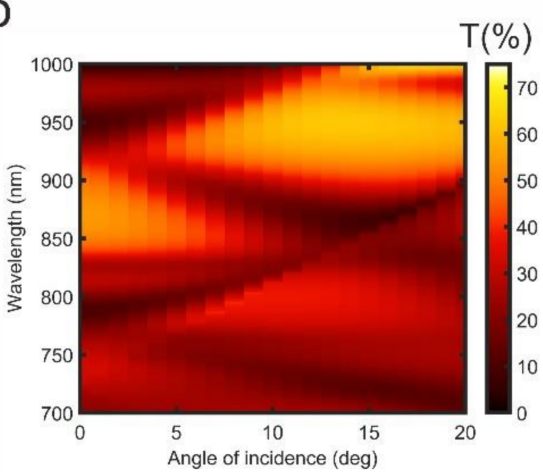

C

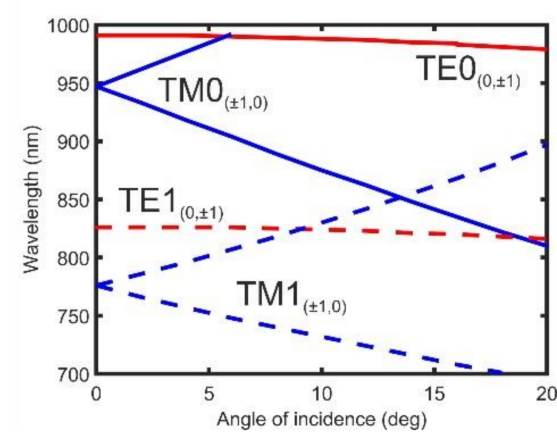

Figure 2. Angle resolved transmission spectra of the sample under p-polarized light excitation. (a) Experimental, (b) numerical, (c) calculated modes dispersion.

The propagating guided optical modes drive the optical and magneto-optical properties of the examined metasurface. To confirm it, we first consider excitation conditions and 
dispersions for both TM and TE guided modes. The phase-matching condition must be met in order to couple incident light with matter via diffraction on Si nanograting:

$$
\beta^{2}=\left(k_{0} \sin (\theta)+2 \pi \frac{m}{d_{x}}\right)^{2}+\left(2 \pi \frac{n}{d_{y}}\right)^{2} .
$$

In Equation (1), $\beta$ is the propagation constant of a mode, $k_{0}=\frac{2 \pi}{\lambda}$ is the free space light wavenumber, $\lambda$ is the free space light wavelength, $\theta$ is the polar incident angle, $d_{x}, d_{y}$ are periods of the structure along the OX and OY directions correspondingly, and $m$ and $n$ are integers which represent the mode order along the OX and OY directions. The propagation constant $\beta$ of the TM or TE guided modes can be calculated using the transcendental equation [36]:

$$
\begin{gathered}
-p_{\{2, N\}} d+\tan ^{-1}\left\{\left(\frac{\epsilon_{2}}{\epsilon_{1}}\right)^{r} \frac{p_{\{1, N\}}}{p_{\{2, N\}}}\right\}+\tan ^{-1}\left\{\left(\frac{\epsilon_{2}}{\epsilon_{3}}\right)^{r} \frac{p_{\{3, N\}}}{\left.p_{\{2, N\}}\right\}}=-N \pi,\right. \\
r=\left\{\begin{array}{c}
0 \text { for TE modes } \\
1 \text { for TM modes }
\end{array}\right.
\end{gathered}
$$

where $p_{\{1, N\}}=\left(\beta^{2}-\epsilon_{1} k_{0}^{2}\right)^{\frac{1}{2}}, p_{\{2, N\}}=\left(\epsilon_{2} k_{0}^{2}-\beta^{2}\right)^{\frac{1}{2}}, p_{\{3, N\}}=\left(\beta^{2}-\epsilon_{3} k_{0}^{2}\right)^{\frac{1}{2}}$, and $\epsilon_{j}$ are dielectric permittivity of the iron garnet film $\left(\epsilon_{2}\right)$ and surrounding claddings $\left(\epsilon_{1}, \epsilon_{3}\right), N$ is the integer that defines the order of the mode (along the OZ direction), and $d$ is the core thickness. In the case of transversal magnetic configuration, Equation (2) does not change for TE modes, but modifies for TM modes:

$$
-p_{\{2, N\}} d+\tan ^{-1}\left\{\left(\frac{\epsilon_{2}}{p_{\{2, N\}}}\right)\left(\frac{p_{\{1, N\}}}{\epsilon_{1}}+\frac{g \cdot \beta}{\epsilon_{2}^{2}}\right)\right\}+\tan ^{-1}\left\{\left(\frac{\epsilon_{2}}{p_{\{2, N\}}}\right)\left(\frac{p_{\{3, N\}}}{\epsilon_{3}}-\frac{g \cdot \beta}{\epsilon_{2}^{2}}\right)\right\}=-N \pi,
$$

where $g$ is a core material gyration constant proportional to its magnetization M.

We calculated the dispersion relation of the modes using Equations (1)-(3). Resonances in the 700-1000 nm spectral region correspond to both TE and TM guided modes (Figure 2c). As previously observed, the $\mathrm{TE}_{(0, \pm 1)}$ and $\mathrm{TE} 1_{(0, \pm 1)}$ (further TE0 and TE1) modes exhibit a weak dependence on incidence angle. On the contrary, resonance positions in transmission spectra of the $\mathrm{TM}_{( \pm 1,0)}$ and $\mathrm{TM} 1_{( \pm 1,0)}$ (further TM0 and TM1) modes are strongly influenced by $\theta$. Notably, the TM0 and TM1 modes spectrally overlap at $850 \mathrm{~nm}$ and $14^{\circ}$ incident angle. The angle-dependent transmittance spectrum simulated numerically using the RCWA method agrees well with the one obtained experimentally (Figure 2b). However, there are minor discrepancies between the calculated positions of the resonances and the ones obtained from experimental data in both transmission and TMOKE spectra. They are caused by the fabrication inaccuracies, which result in a slight difference between geometrical parameters (such as Ce:DyIG thickness and grating period) of the experimental metasurface with their calculated counterparts. Table 1 provides a brief summary of the revealed spectral position features of the resonances.

Table 1. Guided modes' resonant wavelength observed in the transmission spectra.

\begin{tabular}{ccccc}
\hline $\begin{array}{c}\text { Waveguide } \\
\text { Mode }\end{array}$ & $\begin{array}{c}\text { Diffraction } \\
\text { Order }(\mathbf{m}, \mathbf{n})\end{array}$ & $\begin{array}{c}\text { Resonant Wavelength } \\
\text { from Experiment }(\mathbf{n m})\end{array}$ & $\begin{array}{c}\text { Resonant Wavelength } \\
\text { from Simulation }(\mathbf{n m})\end{array}$ & $\begin{array}{c}\text { Resonant Wavelength from } \\
\text { Equations (1)-(3) (nm) }\end{array}$ \\
\hline TE0 & $(0, \pm 1)$ & 985 & 1000 & 991 \\
TM0 & $( \pm 1,0)$ & 935 & 950 & 947 \\
TE1 & $(0, \pm 1)$ & 828 & 933 & 826 \\
TM1 & $( \pm 1,0)$ & 768 & 788 & 776 \\
\hline
\end{tabular}

Electromagnetic energy of the waveguided modes is known to be concentrated inside the core. We numerically simulated the electromagnetic field distribution of optical modes excited by normally incident linearly polarized light to confirm the origin of the resonances. The TM(TE) guided modes possess elliptical polarization with nonzero $\mathrm{E}_{\mathrm{x}}\left(\mathrm{H}_{\mathrm{x}}\right), \mathrm{E}_{\mathrm{z}}\left(\mathrm{H}_{\mathrm{z}}\right)$, and 
$\mathrm{H}_{\mathrm{y}}\left(\mathrm{E}_{\mathrm{y}}\right)$ components. The TM0 guided mode induced by p-polarized light has nonuniform alternate sign $H_{y}$ and $E_{x}$ component distribution along the OX direction and uniform along the OY direction. The situation is inverse for the TE0 one (Figure 3b). There is no alternating sign field behavior along the $\mathrm{OZ}$ direction for both TE0 and TM0 modes.

a

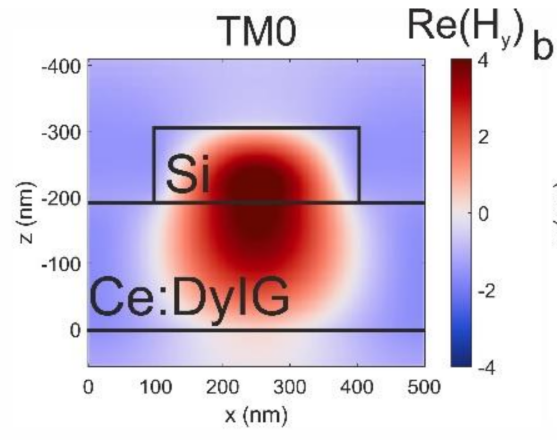

C

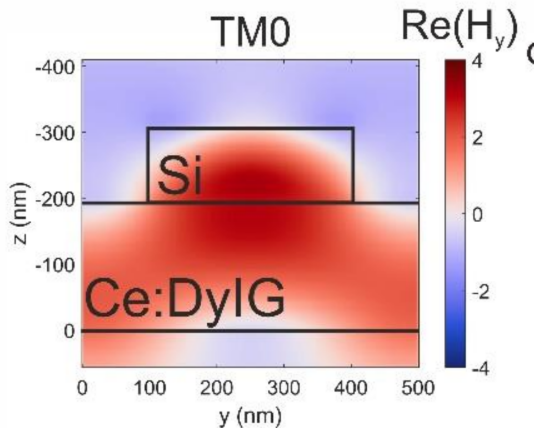

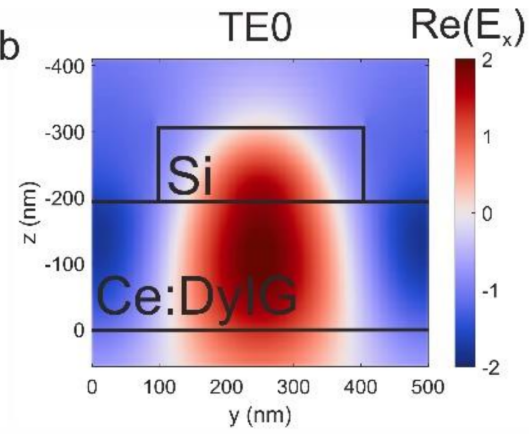

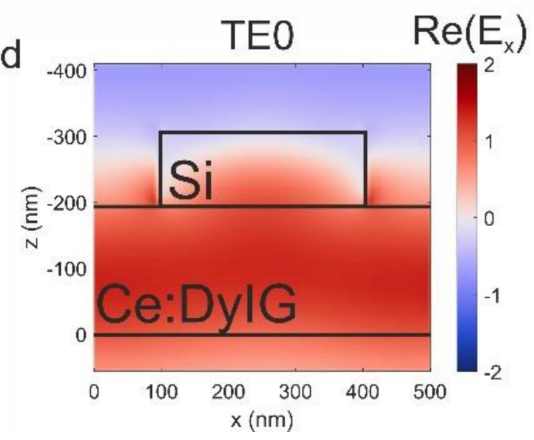

Figure 3. Electromagnetic field distribution of the $\mathrm{TM}_{( \pm 1,0)}(\mathbf{a}, \mathbf{c})$ and $\mathrm{TE}_{(0, \pm 1)}(\mathbf{b}, \mathbf{d})$ modes.

Notably, the TE0 mode electromagnetic field is mainly concentrated inside the garnet film. However, in the TM0 case, the electromagnetic field is slightly squeezed into $\mathrm{Si}$ nanodisk. As a result, the metasurface should be considered as a complex nonuniform waveguide. Furthermore, each Si nanodisk also serves as a scatterer allowing optical and magnetooptical features of the system to be detected in the far field.

The electromagnetic field distribution of the TM1 and TE1 modes along the OX and OY directions is similar to the behavior of TM0 and TE0 modes (see Appendix B, Figure A2). The primary discrepancy is observed along the $\mathrm{OZ}$ direction. While the electromagnetic field distribution of the TM0/TE0 modes is nearly uniform, the TM1/TE1 modes have two antinodes that correspond to the order of the modes.

\subsection{TMOKE Boosted by the Waveguiding Modes}

The transverse magneto-optical Kerr effect (TMOKE) is the magneto-optical intensity effect that can be calculated as follows:

$$
\delta=2 \frac{T(+M)-T(-M)}{T(+M)+T(-M)} \times 100 \%
$$

where $T( \pm M)$ denotes the sample transmittance under antiparallel orientations of the magnetic field (namely, $+M$ and $-M$ ) in the transversal configuration, i.e., for an external magnetic field applied perpendicular to the light incidence plane. TMOKE is typically observed in gyrotropic materials with dissipation [13]. However, in the optical range where iron garnets are relatively transparent for a smooth film, $\delta$ is rarely greater than $\sim 10^{-2 \%}$.

In the transversal magnetization $(M)$ configuration the TM guided modes propagating constant is contributed to by an additional component proportional to magnetization $\beta_{T M}=\beta_{T M}^{0}+\Delta \beta_{T M}(\boldsymbol{M})$ (see Equation (3)). This nonreciprocal contribution to the propagation constant influences transmission spectra, resulting in sharp U-shaped resonances in the TMOKE spectra measured experimentally (Figure 4a). TMOKE spectra calculated nu- 
merically match those obtained experimentally (see Appendix C, Figure A3). The Q-factor $\left(\frac{\lambda}{\Delta \lambda}\right)$ of the TMOKE resonance reaches 85 .
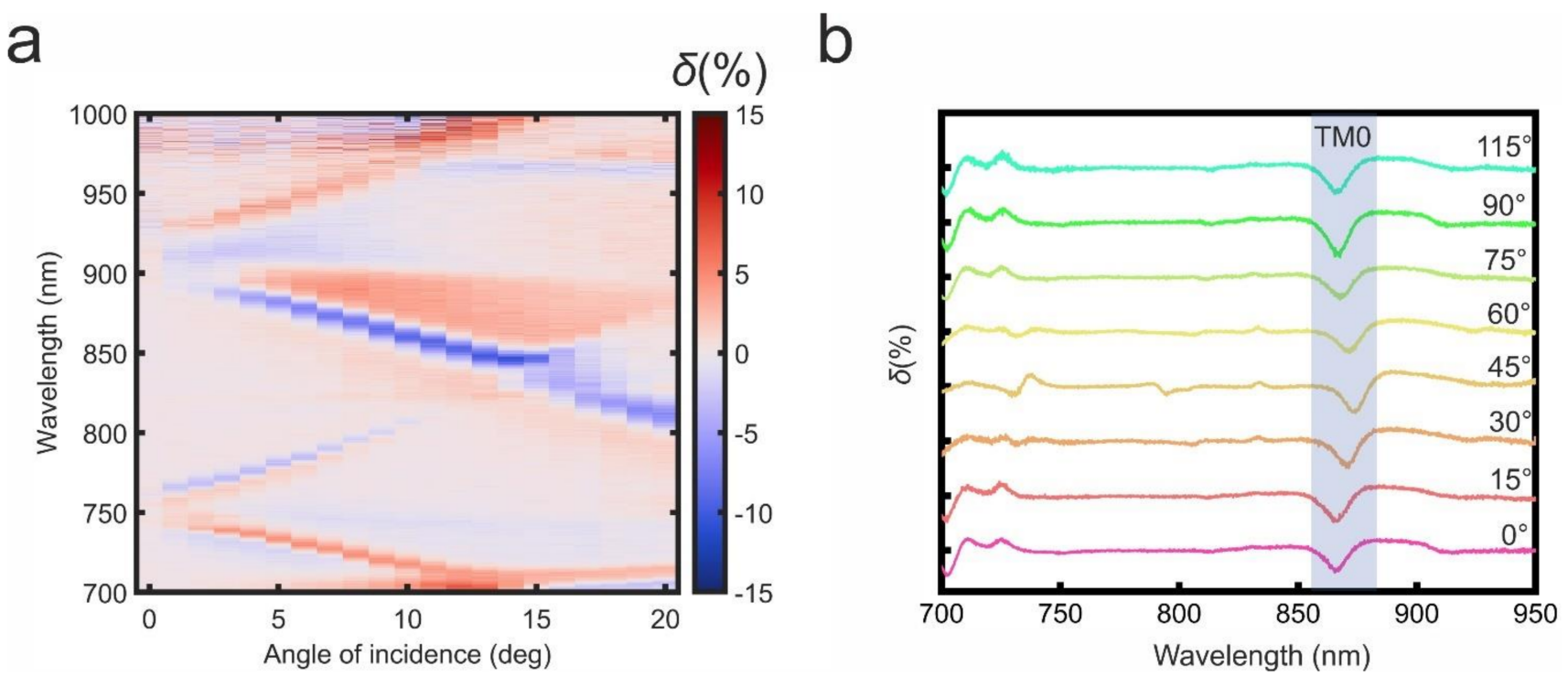

Figure 4. Experimental TMOKE spectra as a function of $\theta$ (a) and for a fixed polar incident angle $\theta=10^{\circ}$ as a function of the azimuth incident angle $\varphi(\mathbf{b})$. All curves in (b) have offsets to clarify the representation.

TMOKE is significantly enhanced in the metasurface under the excitation of TM0 and TM1 modes (Figures $2 \mathrm{c}$ and $4 \mathrm{a}$ ). The greatest $\delta$ increase up to $14 \%$ is observed under the TM0 mode excitation in the angular range $\theta=5 \div 15^{\circ}$ for the wavelengths $850-890 \mathrm{~nm}$ (Figure 4a). This value is about three orders of magnitude larger in comparison to a pristine Ce:DyIG film of the same thickness.

TMOKE potential independence from the orientation of the plane of light incidence with respect to the metasurface is highly demanded for many applications, such as optical modulators, sensors, and magnetometers. Guided by this idea we studied the TMOKE dependence on the azimuth incident angle. We concentrated on the TM0 mode excited at $\sim 860 \mathrm{~nm}$ under $10^{\circ}$ polar incident angle where a pronounced TMOKE enhancement occurs (Figure $4 \mathrm{~b}$ ). Notably, the TMOKE resonance has only a minor spectral deviation when mediated by the TM0 mode, making the proposed magneto-optical metasurface promising for light modulation devices and other applications.

\section{Conclusions}

We designed and fabricated a magneto-optical metasurface for efficient light control via an external magnetic field. It was shown experimentally and numerically that TMOKE is enhanced by about three orders of magnitude in comparison to the pristine Ce:DyIG film due to the TM guided modes excitation. It is critical to note that the TMOKE resonances are unaffected by the orientation of the light incidence plane in relation to the metasurface. The latter feature is important for device applications such as light modulators. Biosensing is another potential application for the suggested gadget. Sharp and prominent resonances of TMOKE may be highly sensitive to the refractive index of the adjacent medium allowing the metasurface to provide sensing functionality. The latter is to be examined in detail in the future. Moreover, as the structure grating is made of $\mathrm{Si}$, the production process is flexible and inclusive of current semiconductor technology.

Author Contributions: Conceptualization, D.O.I., L.B., and V.I.B.; formal analysis, D.M.K.; investigation, D.M.K., S.X., and M.P.M.; methodology, S.X. and M.P.M.; project administration, L.B. and V.I.B.; supervision, D.O.I., L.B., and V.I.B.; writing-original draft, D.M.K., S.X., D.O.I., L.B., and V.I.B.; writing-review and editing, D.M.K., D.O.I., L.B., and V.I.B. All authors have read and agreed to the published version of the manuscript. 
Funding: This research was funded by the Ministry of Science and Higher Education of Russian Federation, Megagrant project N 075-15-2019-1934. S.X. and L.B. are grateful for support from the National Natural Science Foundation of China (NSFC) (grant Nos. 51972044 and 52021001), Ministry of Science and Technology of the People's Republic of China (MOST) (grant Nos. 2016YFA0300802 and 2018YFE0109200), Sichuan Provincial Science and Technology Department (grant Nos. 2019YFH0154 and 2020ZYD015).

Institutional Review Board Statement: Not applicable.

Informed Consent Statement: Not applicable.

Data Availability Statement: The data presented in this study are available on request from the corresponding author.

Conflicts of Interest: The authors declare no conflict of interest.

\section{Appendix A. Optical Properties of Ce:DyIG}

a

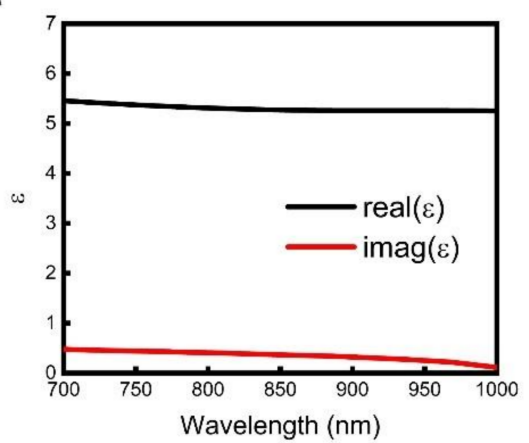

b

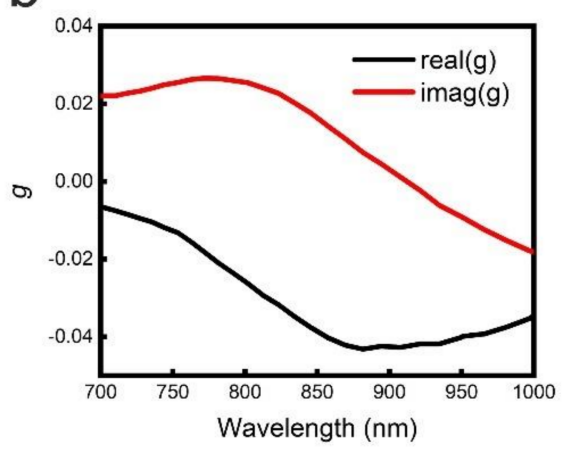

Figure A1. Dielectric permittivity (a) and gyration (b) of Ce:DyIG.

Appendix B. Electromagnetic Field Distribution of the TM1 and TE1 Modes

a

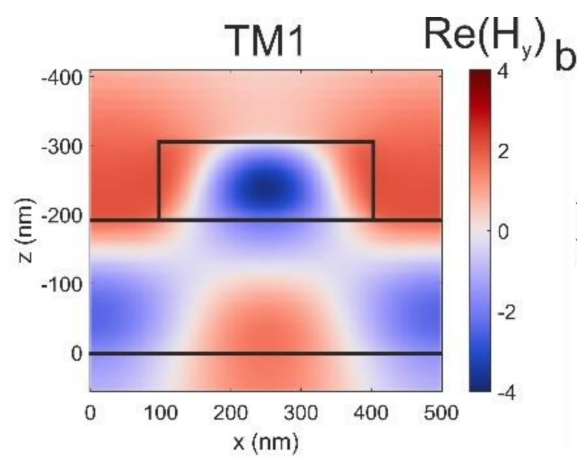

C

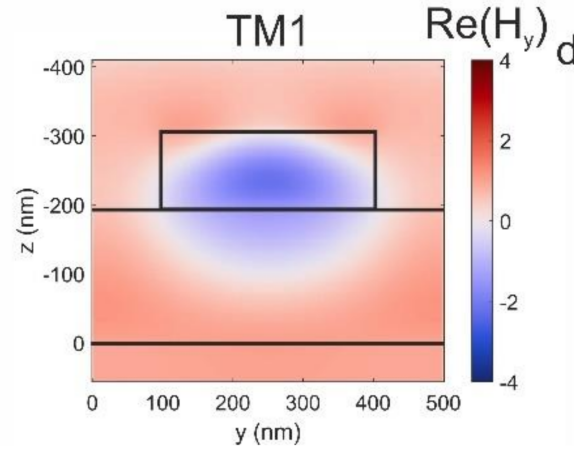

TE1
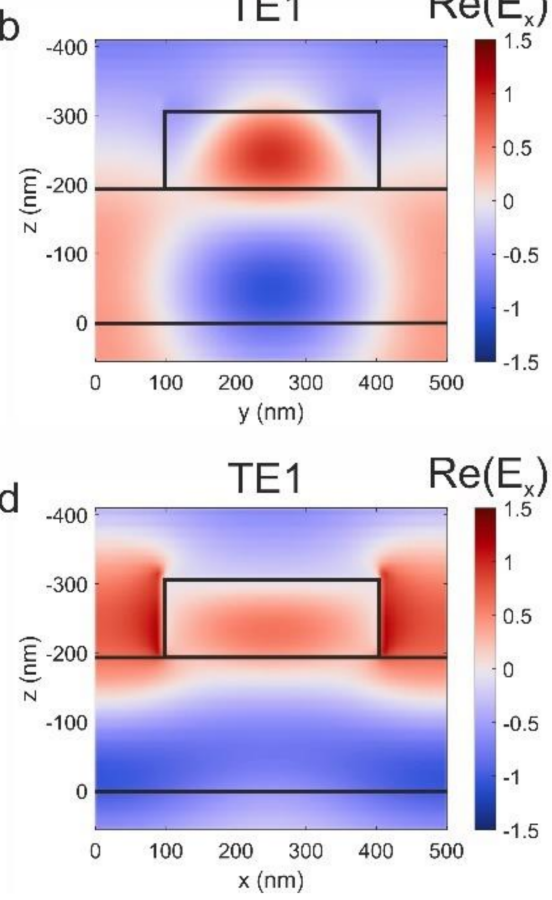

Figure A2. Electromagnetic field distribution of the $\mathrm{TM}_{( \pm 1,0)}(\mathbf{a}, \mathbf{c})$ and $\mathrm{TE} 1_{(0, \pm 1)}(\mathbf{b}, \mathbf{d})$ modes. 


\section{Appendix C. Numerically Calculated Angle-Resolved TMOKE Spectra}

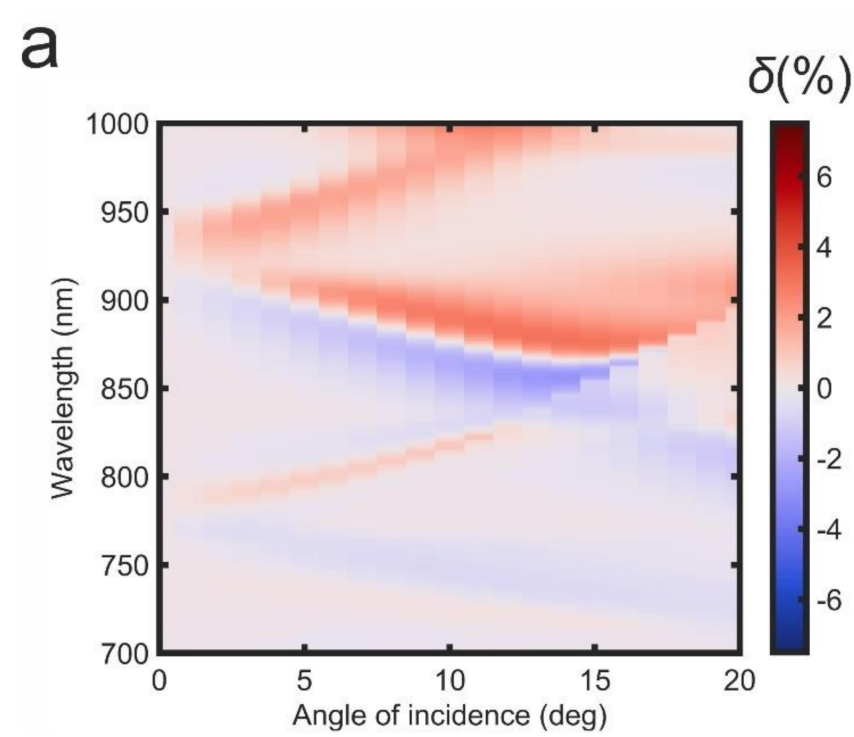

b

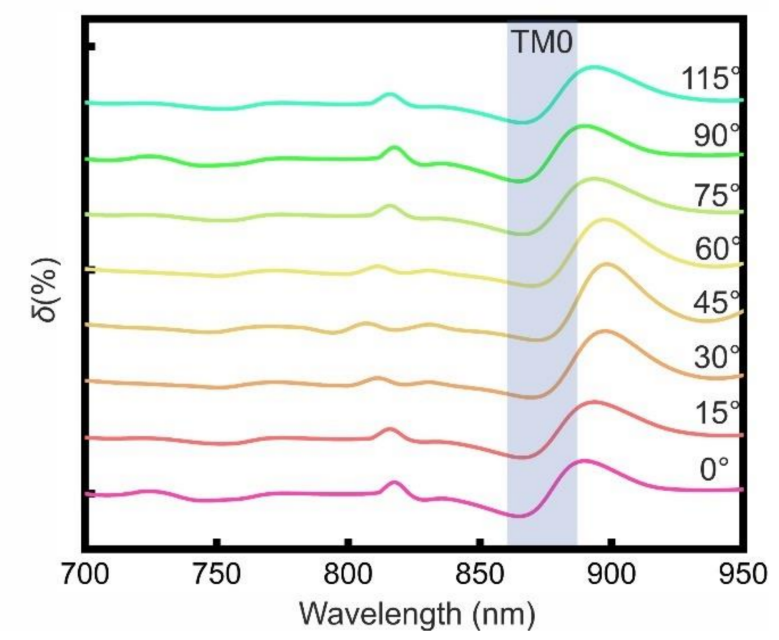

Figure A3. Numerically simulated TMOKE spectra as a function of $\theta$ (a) and for fixed $\theta=10^{\circ}$ as a function of the sample rotation angle $\varphi(\mathbf{b})$. All curves in (b) have offsets to clarify representation.

\section{Appendix D. Schematic of TMOKE Setup}

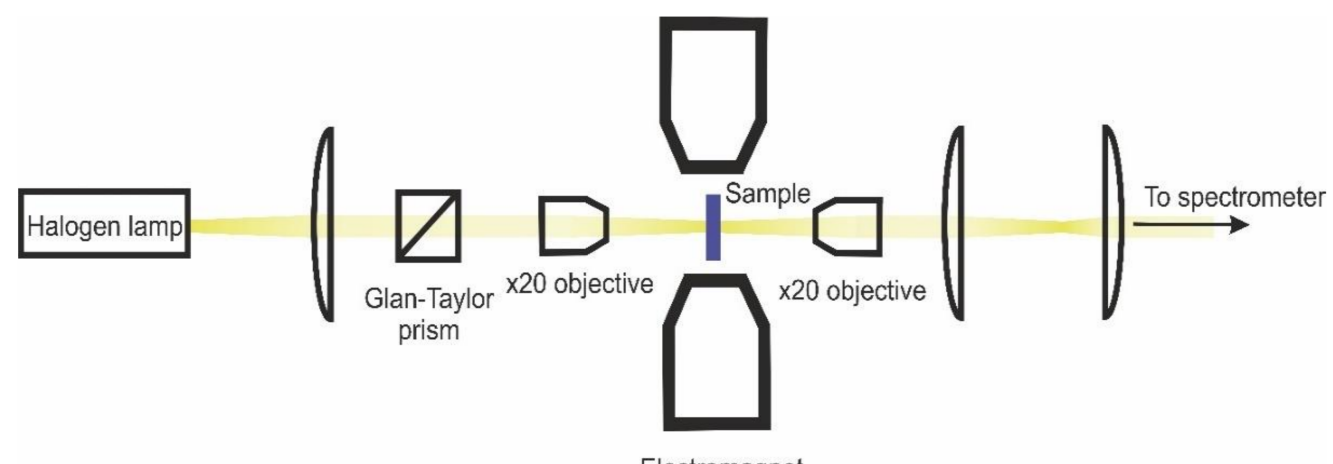

Figure A4. Schematic of TMOKE setup used in the experiment.

\section{References}

1. Silverstone, J.W.; Wang, J.; Bonneau, D.; Sibson, P.; Santagati, R.; Erven, C.; O’Brien, J.L.; Thompson, M.G. Silicon quantum photonics. In Proceedings of the 2016 International Conference on Optical MEMS and Nanophotonics (OMN), Singapore, 31 July-4 August 2016 ; pp. 1-2. [CrossRef]

2. Van Der Sande, G.; Brunner, D.; Soriano, M.C. Advances in photonic reservoir computing. Nanophotonics 2017, 6, 561-576. [CrossRef]

3. Harris, N.C.; Steinbrecher, G.R.; Prabhu, M.; Lahini, Y.; Mower, J.; Bunandar, D.; Chen, C.; Wong, F.N.C.; Baehr-Jones, T.; Hochberg, M.; et al. Quantum transport simulations in a programmable nanophotonic processor. Nat. Photonics 2017, 11, 447-452. [CrossRef]

4. Zasedatelev, A.V.; Baranikov, A.V.; Urbonas, D.; Scafirimuto, F.; Scherf, U.; Stöferle, T.; Mahrt, R.F.; Lagoudakis, P.G. A roomtemperature organic polariton transistor. Nat. Photonics 2019, 13, 378-383. [CrossRef]

5. Bi, L.; Hu, J.; Jiang, P.; Kim, H.; Kim, D.; Onbasli, M.; Dionne, G.; Ross, C. Magneto-Optical Thin Films for On-Chip Monolithic Integration of Non-Reciprocal Photonic Devices. Materials 2013, 6, 5094-5117. [CrossRef]

6. Karki, D.; Stenger, V.; Pollick, A.; Levy, M. Thin-film magnetless Faraday rotators for compact heterogeneous integrated optical isolators. J. Appl. Phys. 2017, 121, 233101. [CrossRef]

7. Krichevsky, D.M.; Tolbin, A.Y.; Dubinina, T.V.; Kosolobov, S.S.; Krasovskii, V.I.; Tomilova, L.G.; Pushkarev, V.E.; Zasedatelev, A.V. Resonant Plasmon-Enhanced Absorption of Charge Transfer Complexes in a Metal-Organic Monolayer. Adv. Opt. Mater. 2021, 9, 2100065. [CrossRef] 
8. Knyazev, G.A.; Kapralov, P.O.; Gusev, N.A.; Kalish, A.N.; Vetoshko, P.M.; Dagesyan, S.A.; Shaposhnikov, A.N.; Prokopov, A.R.; Berzhansky, V.N.; Zvezdin, A.K.; et al. Magnetoplasmonic Crystals for Highly Sensitive Magnetometry. ACS Photonics 2018, 5, 4951-4959. [CrossRef]

9. Borovkova, O.V.; Ignatyeva, D.O.; Sekatskii, S.K.; Karabchevsky, A.; Belotelov, V.I. High-Q surface electromagnetic wave resonance excitation in magnetophotonic crystals for supersensitive detection of weak light absorption in the near-infrared. Photonics Res. 2020, 8, 57. [CrossRef]

10. Ignatyeva, D.O.; Knyazev, G.A.; Kalish, A.N.; Chernov, A.I.; Belotelov, V.I. Vector magneto-optical magnetometer based on resonant all-dielectric gratings with highly anisotropic iron garnet films. J. Phys. D. Appl. Phys. 2021, 54, 295001. [CrossRef]

11. Maccaferri, N.E.; Gregorczyk, K.; de Oliveira, T.V.A.G.; Kataja, M.; van Dijken, S.; Pirzadeh, Z.; Dmitriev, A.; Åkerman, J.; Knez, M.; Vavassori, P. Ultrasensitive and label-free molecular-level detection enabled by light phase control in magnetoplasmonic nanoantennas. Nat. Commun. 2015, 6, 6150. [CrossRef]

12. Berloff, N.G.; Silva, M.; Kalinin, K.; Askitopoulos, A.; Töpfer, J.D.; Cilibrizzi, P.; Langbein, W.; Lagoudakis, P.G. Realizing the classical XY Hamiltonian in polariton simulators. Nat. Mater. 2017, 16, 1120-1126. [CrossRef]

13. Zvezdin, A.K.; Kotov, V.A. Modern Magnetooptics and Magnetooptical Materials; CRC Press: Boca Raton, FL, USA, 1997.

14. Belotelov, V.I.; Akimov, I.A.; Pohl, M.; Kotov, V.A.; Kasture, S.; Vengurlekar, A.S.; Gopal, A.V.; Yakovlev, D.R.; Zvezdin, A.K.; Bayer, M. Enhanced magneto-optical effects in magnetoplasmonic crystals. Nat. Nanotechnol. 2011, 6, 370-376. [CrossRef] [PubMed]

15. Krichevsky, D.M.; Kalish, A.N.; Kozhaev, M.A.; Sylgacheva, D.A.; Kuzmichev, A.N.; Dagesyan, S.A.; Achanta, V.G.; Popova, E.; Keller, N.; Belotelov, V.I. Enhanced magneto-optical Faraday effect in two-dimensional magnetoplasmonic structures caused by orthogonal plasmonic oscillations. Phys. Rev. B 2020, 102, 144408. [CrossRef]

16. Kuzmichev, A.N.; Sylgacheva, D.A.; Kozhaev, M.A.; Krichevsky, D.M.; Shaposhnikov, A.N.; Berzhansky, V.N.; Freire-Fernández, F.; Qin, H.J.; Popova, O.E.; Keller, N.; et al. Influence of the Plasmonic Nanodisk Positions Inside a Magnetic Medium on the Faraday Effect Enhancement. Phys. Status Solidi-Rapid Res. Lett. 2020, 14. [CrossRef]

17. Baryshev, A.V.; Merzlikin, A.M. Tunable plasmonic thin magneto-optical wave plate. J. Opt. Soc. Am. B 2016, 33, 1399. [CrossRef]

18. Kalish, A.N.; Komarov, R.S.; Kozhaev, M.A.; Achanta, V.G.; Dagesyan, S.A.; Shaposhnikov, A.N.; Prokopov, A.R.; Berzhansky, V.N.; Zvezdin, A.K.; Belotelov, V.I. Magnetoplasmonic quasicrystals: An approach for multiband magneto-optical response. Optica 2018, 5, 617. [CrossRef]

19. Belotelov, V.I.; Zvezdin, A.K. Magnetooptics and extraordinary transmission of the perforated metallic films magnetized in polar geometry. J. Magn. Magn. Mater. 2006, 300, e260-e263. [CrossRef]

20. Khramova, A.E.; Ignatyeva, D.O.; Kozhaev, M.A.; Dagesyan, S.A.; Berzhansky, V.N.; Shaposhnikov, A.N.; Tomilin, S.V.; Belotelov, V.I. Resonances of the magneto-optical intensity effect mediated by interaction of different modes in a hybrid magnetoplasmonic heterostructure with gold nanoparticles. Opt. Express 2019, 27, 33170. [CrossRef] [PubMed]

21. Maccaferri, N.; Zubritskaya, I.; Razdolski, I.; Chioar, I.-A.; Belotelov, V.; Kapaklis, V.; Oppeneer, P.M.; Dmitriev, A. Nanoscale magnetophotonics. J. Appl. Phys. 2020, 127, 080903. [CrossRef]

22. Voronov, A.A.; Karki, D.; Ignatyeva, D.O.; Kozhaev, M.A.; Levy, M.; Belotelov, V.I. Magneto-optics of subwavelength all-dielectric gratings. Opt. Express 2020, 28, 17988. [CrossRef] [PubMed]

23. Ignatyeva, D.O.; Karki, D.; Voronov, A.A.; Kozhaev, M.A.; Krichevsky, D.M.; Chernov, A.I.; Levy, M.; Belotelov, V.I. All-dielectric magnetic metasurface for advanced light control in dual polarizations combined with high-Q resonances. Nat. Commun. 2020, 11 [CrossRef]

24. Chernov, A.I.; Kozhaev, M.A.; Ignatyeva, D.O.; Beginin, E.N.; Sadovnikov, A.V.; Voronov, A.A.; Karki, D.; Levy, M.; Belotelov, V.I. All-Dielectric Nanophotonics Enables Tunable Excitation of the Exchange Spin Waves. Nano Lett. 2020, 20, 5259-5266. [CrossRef]

25. Bsawmaii, L.; Gamet, E.; Royer, F.; Neveu, S.; Jamon, D. Longitudinal magneto-optical effect enhancement with high transmission through a 1D all-dielectric resonant guided mode grating. Opt. Express 2020, 28, 8436. [CrossRef]

26. Royer, F.; Varghese, B.; Gamet, E.; Neveu, S.; Jourlin, Y.; Jamon, D. Enhancement of Both Faraday and Kerr Effects with an All-Dielectric Grating Based on a Magneto-Optical Nanocomposite Material. ACS Omega 2020, 5, 2886-2892. [CrossRef]

27. Dong, D.; Liu, Y.; Fu, Y. Enhancing the Faraday rotation of monolayer black phosphorus by the optical Tamm state at the photonic crystal interface. Appl. Opt. 2020, 59, 9607. [CrossRef] [PubMed]

28. Koerdt, C.; Rikken, G.L.J.A.; Petrov, E.P. Faraday effect of photonic crystals. Appl. Phys. Lett. 2003, 82, 1538-1540. [CrossRef]

29. Kim, I.; Kim, W.S.; Kim, K.; Ansari, M.A.; Mehmood, M.Q.; Badloe, T.; Kim, Y.; Gwak, J.; Lee, H.; Kim, Y.K.; et al. Holographic metasurface gas sensors for instantaneous visual alarms. Sci. Adv. 2021, 7. [CrossRef] [PubMed]

30. Zhou, H.; Sain, B.; Wang, Y.; Schlickriede, C.; Zhao, R.; Zhang, X.; Wei, Q.; Li, X.; Huang, L.; Zentgraf, T. Polarization-Encrypted Orbital Angular Momentum Multiplexed Metasurface Holography. ACS Nano 2020, 14, 5553-5559. [CrossRef]

31. Lawrence, M.; Barton, D.R.; Dionne, J.A. Nonreciprocal Flat Optics with Silicon Metasurfaces. Nano Lett. 2018, 18, 1104-1109. [CrossRef] [PubMed]

32. Cordaro, A.; Kwon, H.; Sounas, D.; Koenderink, A.F.; Alù, A.; Polman, A. High-Index Dielectric Metasurfaces Performing Mathematical Operations. Nano Lett. 2019, 19, 8418-8423. [CrossRef]

33. Grann, E.B.; Pommet, D.A.; Moharam, M.G.; Gaylord, T.K. Formulation for stable and efficient implementation of the rigorous coupled-wave analysis of binary gratings. J. Opt. Soc. Am. A 1995, 12, 1068-1076.

34. Li, L. Fourier modal method for crossed anisotropic gratings with arbitrary permittivity and permeability tensors. J. Opt. A Pure Appl. Opt. 2003, 5, 345-355. [CrossRef] 
35. Palik, E.D. Handbook of Optical Constants of Solids; Elsevier: Amsterdam, The Netherlands, 1985; ISBN 9780080547213.

36. Yariv, A.; Yeh, P. Optical Waves in Crystals; Wiley: New York, NY, USA, 1984. 